\title{
Measurement of Local Atomic Displacements Reveals Interaction of Au Nanocrystals with Rutile $\left(\mathrm{TiO}_{2}\right)$ Surface Steps
}

\author{
Wenpei Gao ${ }^{1,2}$ and Jian-Min Zuo ${ }^{1,2}$ \\ 1. Dept of Materials Science and Engineering, University of Illinois, Urbana-Champaign, IL 61801 \\ 2. Frederick Seitz Materials Research Laboratory, University of Illinois, Urbana-Champaign, IL \\ 61801
}

Advances in atomic resolution electron imaging now promise a detailed understanding of nanostructures and their interfaces, as well as defects. A major challenge in nanostructure characterization is the study of $3 \mathrm{D}$ interfaces that deviate significantly from a flat, 2D, interface. Here, we report a significant progress in the study of 3D interfaces by examining local displacements of Au atoms near surface steps on rutile $\left(\mathrm{TiO}_{2}\right)(110)$ surface. The study was carried out using atomic resolution Z-contrast imaging. The local atomic structure and strain is determined using a recently developed lattice analysis method, called TeMA, based on the analysis of atomic resolution images using template matching [1].

For the experiment, $\mathrm{Au} \mathrm{NCs}$ on vicinal $\mathrm{TiO}_{2}$ surfaces are prepared using the previously reported synthesis technique [2] that enables high resolution STEM investigation. Au nanoparticles about 2 5 nm in diameters, were first deposited onto the single crystal $\mathrm{TiO} 2$ substrate, pre-thinned and annealed in air, by e-beam evaporation deposition at room temperature. Then the sample was annealed in air at selected temperatures to promote epitaxy. A JEOL 2200FS STEM with a CEOS probe Cs corrector and annular dark-field (ADF) detector was used for atomic resolution Z-contrast imaging. The microscope was operated at $200 \mathrm{kV}$.

Figure 1 shows a Z-contrast image of the Au NCs on $\mathrm{TiO}_{2}$ (110) surface steps. The image was taken along the $\mathrm{TiO} 2$ [001] and $\mathrm{Au}$ [110] zone axis directions. Both atomic columns in the Au NCs and $\mathrm{Ti}$ columns in $\mathrm{TiO}_{2}$ are clearly resolved because of the approximately epitaxial relationship categorized as $\mathrm{Au}(111)[-110] \| \mathrm{TiO}_{2}(110)[001]$.. The brighter contrast of Au compared to the duller contrast of $\mathrm{Ti}$ is due to the Z-contrast difference obtained using a high inner cutoff angle for the ADF detector.

To measure atomic positions, first an image of the individual atomic column is obtained by spatial averaging over a group of atomic columns in the image. Then, this averaged image is used as the template to detect individual atomic columns and locate their positions with sub-pixel precision. The positions are then used to calculate the projected atomic displacements (in 2D) at atomic resolution using a selected region of the $\mathrm{Au} \mathrm{NC}$ as reference. The scan noise is corrected using the substrate $\left(\mathrm{TiO}_{2}\right)$ as the reference. After the correction, the substrate $\left(\mathrm{TiO}_{2}\right)$ lattice is $\mathrm{a}=\mathrm{b}=3.25 \AA, \alpha=90^{\circ}$, and the $2 \mathrm{D} \mathrm{Au}$ lattice along $\mathrm{Au}$ [001] zone axis in average is measured as: $\mathrm{a}=2.52 \AA, b=2.48 \AA, \alpha=109.6^{\circ}$. For comparison, the standard values for the $2 \mathrm{D}$ gold lattice along [001] zone axis is: $\mathrm{a}=\mathrm{b}=2.50 \AA, \alpha=109.5^{\circ}$.

Figure 2 indicates the displacement of atomic columns from the average lattice sites (indicated by small circles); the displacement vector of each atomic column is displayed by a line for both Au and Ti. The amount of displacement is magnified by a factor of 4 for the image. The line originates from the average lattice site and ends at the location of the atomic column determined from the Z-contrast image. Deformation can be seen directly in the image for the interfacial atomic columns near the surface steps. Large displacements of $0.65 \AA$ are seen at the interfacial Au layer. Here, atoms move horizontally on the 
terrace and displace vertically near the steps. The interfacial displacement reduces in atomic layers away from the interface, especially for these at 3 layers away or more. Displacement of Ti atoms is also seen, but small compared to the position shift of Au atoms in the NC [3][4].

\section{References:}

[1] J.M. Zuo, A.B. Shah, H. Kim, Y.F. Meng, W.P. Gao, J.L. Rouviere, Ultramicr. 136, 50 (2013)

[2] S. Sivaramakrishnan, J.G. Wen, M.E. Scarpelli, et. al., Phys. Rev. B 82, 195421 (2010)

[3] W. P. Gao, A. Choi and J. M. Zuo, Surf. Sci., Accepted (2014)

[4] The work reported here is supported by the NSF Grant No. DMR 0449790 and AC-STEM was carried out in the Frederick Seitz Materials Research Laboratory Central Facilities, University of Illinois.

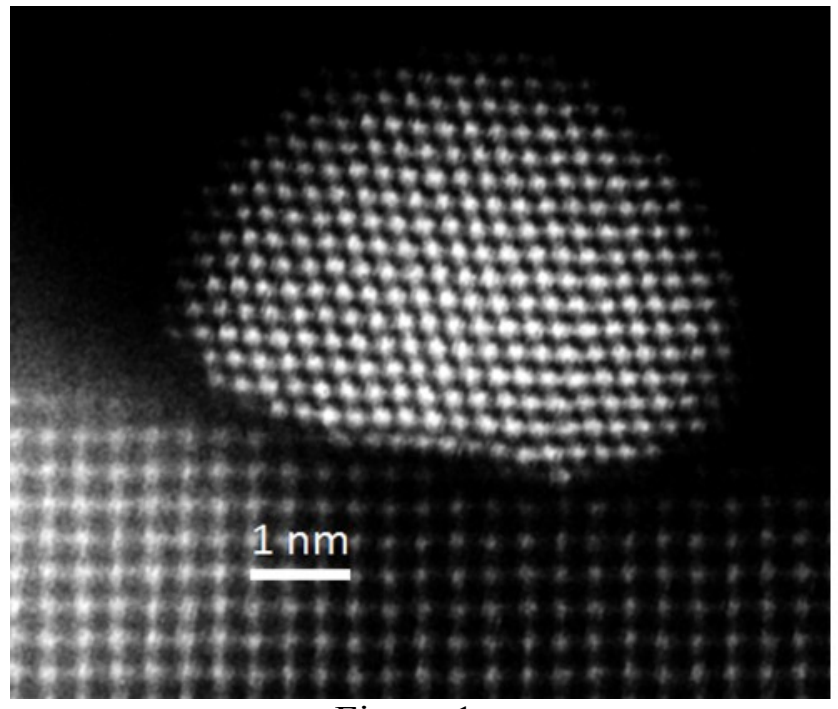

Figure 1

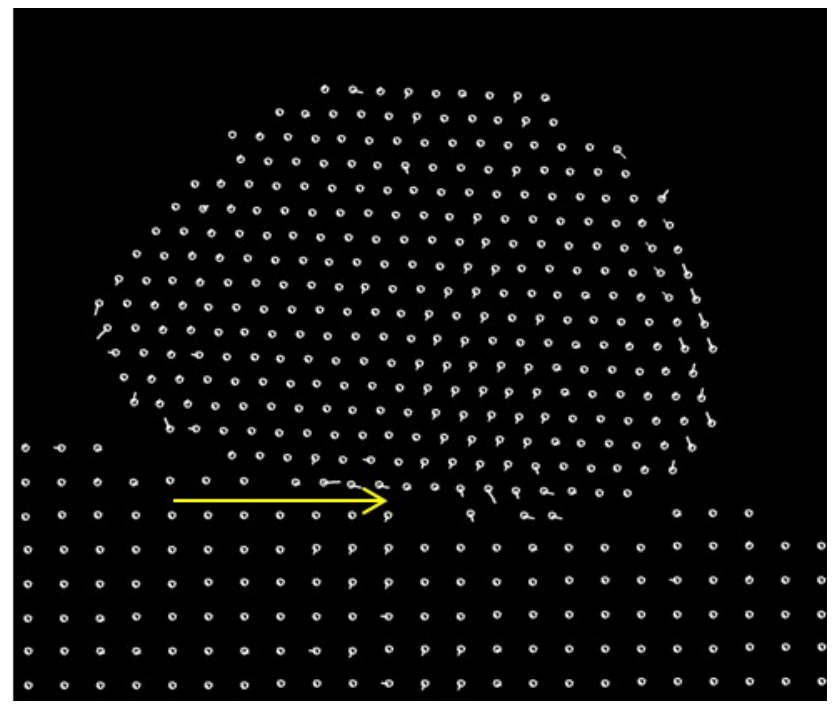

Figure 2

Figure 1, Atomic-resolution HAADF-STEM images of Au NCs of different sizes in approximately epitaxial relationship of $\mathrm{Au}(11-1)_{[112]}|| \mathrm{TiO}_{2}(110)_{[1-10]}$. The images were recorded along the $\mathrm{TiO}_{2}[001]$, or $\mathrm{Au}$ [110], zone axis. The $\mathrm{TiO}_{2}$ appears in a square lattice.

Figure 2, Au atomic displacements measured from the atomic resolution image shown in Figure 1. The interface is indicated by the yellow arrow. The Au atom positions in an undistorted lattice are marked by circles, while the lines indicate the amount and direction of measured atomic displacements (for details, see text). 